\title{
BMJ A randomised controlled non-inferiority Open trial of primary care-based facilitated access to an alcohol reduction website (EFAR-FVG): the study protocol
}

To cite: Struzzo P, Scafato E, McGregor R, et al. A randomised controlled noninferiority trial of primary care-based facilitated access to an alcohol reduction website (EFAR-FVG): the study protocol. BMJ Open 2013;3:e002304.

doi:10.1136/bmjopen-2012002304

- Prepublication history for this paper are available online. To view these files please visit the journal online (http://dx.doi.org/10.1136/ bmjopen-2012-002304).

Received 6 November 2012 Revised 11 January 2013 Accepted 21 January 2013

This final article is available for use under the terms of the Creative Commons Attribution Non-Commercial 2.0 Licence; see http://bmjopen.bmj.com

For numbered affiliations see end of article

Correspondence to Dr Pierluigi Struzzo; pierluigi.struzzo@uniud.it

\section{ABSTRACT}

Introduction: There is a strong body of evidence demonstrating the effectiveness of brief interventions by primary care professionals for risky drinkers. However, implementation levels remain low because of time constraints and other factors. Facilitated access to an alcohol reduction website offers primary care professionals a time-saving alternative to standard face-to-face intervention, but it is not known whether it is as effective.

Methods and analysis: A randomised controlled non-inferiority trial for risky drinkers comparing facilitated access to a dedicated website with standard face-to-face brief intervention to be conducted in primary care settings in the Region of Friuli Giulia Venezia, Italy. Adult patients will be given a leaflet inviting them to log on to a website to complete the Alcohol Use Disorders Identification Test (AUDIT-C) alcohol screening questionnaire. Screen positives will be requested to complete an online trial module including consent, baseline assessment and randomisation to either standard intervention by the practitioner or facilitated access to an alcohol reduction website. Follow-up assessment of risky drinking will be undertaken online at 1 month, 3 months and 1 year using the full AUDIT questionnaire. Proportions of risky drinkers in each group will be calculated and non-inferiority assessed against a specified margin of $10 \%$. Assuming a reduction of $30 \%$ of risky drinkers receiving standard intervention, 1000 patients will be required to give $90 \%$ power to reject the null hypothesis.

Ethics and dissemination: The protocol was approved by the Isontina Independent Local Ethics Committee on 14 June 2012. The findings of the trial will be disseminated through peer-reviewed journals, national and international conference presentations and public events involving the local administrations of the towns where the trial participants are resident.

Registration details: Trial registration number NCT: 01638338.

\section{ARTICLE SUMMARY}

Article focus

- Is the website-facilitated access to alcohol brief Intervention (BI) as good as face-to-face $\mathrm{Bl}$ ?

- Is primary care the right setting to promote internet usage?

Key messages

- Risky drinking is an important health issue.

- General practices are too busy to provide BI on alcohol.

Strengths and limitations of this study

- A new, widespread tool is proposed to reduce risky drinking.

- If not effective, this study will promote BI among general practitioners.

- The domestic use of computers is not widespread in Italy, and community involvement might be important.

\section{BACKGROUND}

Hazardous alcohol consumption is a significant public health problem, with an estimated $3.8 \%$ of all global deaths and $4.6 \%$ of global disability-adjusted life years lost attributable to alcohol. ${ }^{1}$ The European Union (EU) is the heaviest drinking region in the world, drinking an average of 11 litres of pure alcohol per adult each year. ${ }^{2}$ In Region Friuli Venezia Giulia, risky alcohol consumption varies between $23.2 \%$ and $37.4 \%$ of the general population, being more significant in young adults (18-24 years). ${ }^{3-6}$ There is strong evidence that screening and brief interventions (SBIs) are effective in reducing both alcohol consumption and the harms associated with hazardous drinking. ${ }^{7}$ However, in primary care, less than $10 \%$ of hazardous and harmful drinkers are 
identified and less than $5 \%$ of those who could benefit are offered BIs. ${ }^{8}$ One of the principal reasons for this is that conventional delivery of BI can add up to 15 min to the primary consultation. In Italy, research on the topic of SBI in general practice is scarce and although there is some evidence about general practitioners' (GPs') knowledge and attitudes, ${ }^{9}$ no evidence exists on the extent to which SBIs are currently implemented.

The provision of facilitated access by primary care professionals to an alcohol reduction website could significantly increase BI rates by offering a time-saving alternative to face-to-face intervention. A review of trials of computer-based interventions in college drinkers found them to be more effective than no treatment and as effective as alternative treatment approaches. The recent online trial of Down Your Drink (http://www. downyourdrink.org.uk/) indicated potentially significant reductions in alcohol consumption and risky drinking behaviours in subjects who used the trial websites, ${ }^{10}$ and a number of initiatives are underway to test the acceptability of this approach. ${ }^{11}$ The EU-funded ODHIN trial currently underway in five European countries is designed to determine the impact of facilitated access on levels of implementation of BIs by primary care practitioners. The DYD website has also been deployed in two NHS Primary Care Trust settings in London, including the automated baseline assessment of alcohol consumption and alcoholrelated problems together with facilitated access by primary care professionals. The initial findings suggest that facilitated access is both feasible and attractive in primary care settings, but evidence is required to establish whether it is as effective as standard intervention.

\section{METHODS AND ANALYSIS}

\section{Trial design}

Effectiveness of primary care based Facilitated Access to alcohol Reduction website - a non-inferiority randomized controlled trial-Friuli Venezia Giulia (EFAR-FVG) is a primary care-based randomised controlled noninferiority trial comparing facilitated access to a dedicated website for risky drinkers with standard face-to-face BI. With the exception of the reference intervention, all components of the trial will be delivered online to patients who have been given a unique trial log-on number by their general practice. Access will be through the healthy lifestyle portal of the official website of the Region of Friuli Venezia Giulia.

\section{The trial website}

The website is an Italian language online facility which includes modules for all the key trial components including screening, consent, assessment, randomisation and follow-up. It also incorporates the alcohol reduction website for the patients in the experimental group. The site has been adapted from the www.DownYourDrink.org. uk (DYD) website developed for the DYD-randomised controlled trial (RCT) trial. ${ }^{10}$ Details of the DYD website and the psychological theory which has underpinned its development have been reported elsewhere. ${ }^{12}$ Country-specific information such as the recommended guidelines for alcohol intake, definitions of standard drinks and alcohol-related laws will be included. The EFAR-FVG trial website will additionally incorporate a menu-driven facility to enable the GPs to personalise the automated patient messages by adding photographs of themselves and audio/video recorded messages.

\section{Practices}

The trial will be conducted in primary care settings in the Italian region of Friuli Venezia Giulia, and general practices will be invited by email and letter to participate in the study. Those expressing an interest in the trial and a willingness to actively distribute the brochure inviting patients to $\log$ on to the trial website will be considered for inclusion in the study. Preference will be given to practices with at least 1000 registered patients and a practice nurse and/or receptionist.

\section{Training}

All participating GPs will attend a 1-day training event including an overview of the EFAR-FVG trial and interactive sessions on the delivery of face-to-face BI for risky drinkers. Participants will be encouraged to familiarise themselves with the trial website and will have the opportunity to use the menu-driven facility to create their own tailored patient messages.

\section{Patient eligibility}

All patients aged 18 or over who attend the participating practices during the study period will be eligible for the trial. Patients over 80 and those suffering from severe psychiatric disorder, serious visual impairment or terminal illness will be excluded from the study, as will those judged to have inadequate command of the Italian language.

\section{Screening}

Eligible patients will be encouraged by their GP or another staff member to access a specially designed healthy lifestyle website and will be given a trial brochure providing a unique access number which will enable them to $\log$ on. Once online, they will be asked to complete the three-question short Alcohol Use Disorders Identification Test (AUDIT-C) ${ }^{13}$ and to provide agreement to the result of the test to be sent to their practice. For the purposes of the trial, cut points of 4 for women and 5 for men will be used. Patients screening below the cut points will receive an online message advising that their responses indicate that their stated drinking patterns fall within the guidelines for sensible drinking and will be encouraged to maintain their current drinking patterns. Those scoring at or above the cut points will receive a personalised online message from their GP advising that their stated drinking patterns indicate that they are at risk from their drinking and inviting them to take part in the study. 


\section{Consent}

Screen positive subjects will be asked to complete an online form confirming that they do not meet any of the exclusion criteria and then be invited to complete the online consent module. This provides information about the trial and what will be requested of them as participants. Those providing consent will be invited to complete the online baseline assessment.

\section{Baseline assessment}

The baseline assessment includes the following:

1. A demographic questionnaire seeking information on age, gender, level of education and occupation.

2. The 10-question AUDIT validated Italian version. ${ }^{14}$

3. D5-EQ D5 the quality-of-life questionnaire, validated Italian version. ${ }^{15}$

\section{Randomisation}

Those completing the baseline questionnaires will undergo automated online randomisation.

\section{Experimental group-facilitated access to the alcohol reduction website}

Those in the experimental group will be taken to the introductory page containing a personalised online message from their GP with tailored feedback about their responses to the alcohol questionnaires. Personalised online messages from their GP will inform patients of the importance of adopting healthy drinking choices, and will encourage them to spend at least 15 min engaging with the alcohol reduction website in the first instance. Patients will receive an email 1 week later encouraging them to log on again. They will also be asked online to review their alcohol consumption and be invited to discuss their website experience when they next see their GP.

\section{Reference group-face-to-face BI}

Patients allocated to the standard intervention group will be invited to check a box online which will automatically generate an email to their practice requesting a GP appointment for a face-to-face BI within the next 710 days. At the appointment, the patient will receive an intervention that will be based on the brief motivational interview $^{16}$ :

1. Assessment of the motivation to change the risky behaviour;

2. Assessment of the stage of change;

3. Advice-explicit advice on changing drinking behaviour;

4. Empathy-role of counsellor is important;

5. Capacity building-instilling optimism that goals are achievable.

Non-attenders will be offered up to three additional appointments in order to optimise their intervention rates.
Follow-up assessment

Follow-up will take place at 1, 3 and 12 months after randomisation, and each assessment will consist of the following:

1. The 10-question AUDIT validated Italian version.

2. D quality of life questionnaire, validated Italian version 5-The EQ.

Every effort will be made to optimise response rates. In the first instance, each patient in the trial will receive an automated email requesting them to login and complete the assessment questionnaires. Failure to do so will result in further emails at 1-week and 2-week intervals. Persistent failure will be notified to the patient's GP who will contact them by letter, phone or in person in order to ensure that their assessment is completed.

\section{Statistical analysis}

The principal outcome measure will be the proportion of risky drinkers as classified by responses to the AUDIT-10 question scale at 3 months following randomisation.

In order to assess the non-inferiority of facilitated access compared with face-to-face BI, the proportions of risky drinkers in each group will be computed and compared using generalised non-linear mixed models accounting for general practices as random effects. Facilitated access will be deemed not inferior to face-to-face treatment at a one-sided $\alpha$ of $2.5 \%$ if the difference between the proportions of risky drinkers in the facilitated access group and the face-to-face BI group is below a specified margin of non-inferiority of $10 \%$. Assuming a reduction of $30 \%$ in the proportion of risky drinkers in the face-to-face intervention group and allowing for an overall attrition of $10 \%$ of patients in the trial, it is calculated that 500 patients will be required in each group to give the trial $90 \%$ power (1- $\beta$ ) to reject the null hypothesis that facilitated access is inferior to face-to face intervention. All analyses will be described in a detailed statistical analysis plan which will be completed before unblinding and database lock.

\section{Author affiliations}

${ }^{1}$ Region Friuli Venezia Giulia, Regional Centre for the Training in Primary Care, Monfalcone, Italy

${ }^{2}$ Istituto Superiore di Sanità, WHO Collaborating Centre for Research and Health Promotion on Alcohol and Alcohol-Related Health Problems,

Osservatorio Nazionale Alcol, Centro Nazionale di Epidemiologia, Sorveglianza e Promozione della Salute, Rome, Italy

${ }^{3}$ Codeface Ltd, Hove, UK

${ }^{4}$ Movimento Giotto, Bologna, Italy

${ }^{5}$ Department of Primary Care and Population Health, University College London, London, UK

${ }^{6}$ National Institute of Health Research Clinical Research Networks, University of Leeds, Leeds, UK

Contributors PS and PW are the principal investigators who designed the study and drafted the article. ES, RM, HL, RDV, LV and CT have made substantial contributions to the conception and design of the project. NF has been involved in drafting the manuscript or revising it critically for important intellectual content. All authors read and approved the final manuscript. 
Funding This work is jointly supported by the Italian Ministry of Health and by the Regional school for the training in Primary Care of the Region Friuli-Venezia Giulia, Italy (grant number D25E12002900003).

Competing interests None.

Ethics approval Ethical Committee of the Azienda Sanitaria 4 Medio Friuli, Udine, IT. The protocol was approved on 14 June 2012 by the Independent Local Ethics Committee for Clinical Research of the Health Services Agency No 2 Isontina, Italy.

Provenance and peer review Not commissioned; externally peer reviewed.

Data management and security Regular checks of the quality of the data will be carried out under the supervision of the research team. Data files generated by the patients' interactions with the alcohol reduction website will be stored securely on servers in accordance with EU regulations. The only identifiers will be the unique login number. The files generated by the practices linking the unique login numbers to the patient identifiers will be stored securely along with other clinical data in the practice and be accessible only to practice staff.

\section{REFERENCES}

1. Rehm J, Mathers C, Popova S, et al. Global burden of disease and injury and economic cost attributable to alcohol use and alcohol-use disorders. Lancet 2009;373:2223-33.

2. Anderson P, Baumberg B. Alcohol in Europe: a public health perspective. London: Institute of Alcohol Studies, 2006

3. PASSI: progressi delle aziende sanitarie per la salute in Italia. December 2011 data. http://www.epicentro.iss.it/passi (accessed 22 Mar 2012).

4. Simoes EJ, Mariotti S, Rossi A, et al. The Italian health surveillance (SiVeAS) prioritization approach to reduce chronic disease risk factors. Int J Public Health 2012;57:719-33.
5. Rehm J, Scafato E. Indicators of alcohol consumption and attributable harm for monitoring and surveillance in European Union countries. Addiction 2011;106(Suppl 1):4-10.

6. Drummond C, Gual A, Goos C, et al. Identifying the gap between need and intervention for alcohol use disorders in Europe. Addiction 2011;106(Suppl 1):31-6.

7. Kaner EFS, Dickinson HO, Beyer FR, et al. Effectiveness of brief alcohol interventions in primary care populations. Cochrane Database Syst Rev 2007(2). doi: 10.1002/14651858.CD004148. pub3

8. Anderson P, Chisholm D, Fuhr DC. Effectiveness and cost-effectiveness of policies and programmes to reduce the harm caused by alcohol. Lancet 2009;373:2234-46.

9. Struzzo P, Gianmoena B, Kodilija R. The attitude and knowledge of Italian family doctors in respect to early identification and brief intervention on alcohol \& tobacco: a controlled study. Family medicine on-line. http://www.priory.com/fam/italgp.htm (accessed 22 Mar 2012).

10. Wallace $\mathrm{P}$, Murray E, McCambridge $\mathrm{J}$, et al. On-line randomized controlled trial of an Internet based psychologically enhanced intervention for people with hazardous alcohol consumption. PLOS ONE 2011;6:e14740.

11. http://www.odhinproject.eu/

12. Linke S, McCambridge J, Khadjesari Z, et al. Development of a psychologically enhanced interactive online intervention for hazardous drinking. Alcohol Alcohol 2008;43:669-74.

13. Struzzo P, De Faccio S, Moscatelli E. Identificazione precoce dei bevitori a rischio in Assistenza Primaria in Italia:o ed adattamento del questionario AUDIT al contesto italiano e verifica dell' efficacia d'uso dello short-AUDIT test nel contesto nazionale di assistenza primaria: uno studio di validazione interna. Boll delle Farmacodipendenze e Alcolismo 2006;XXIX:20-5.

14. Saunders JB, Aasland OG, Babor TF, et al. Development of the Alcohol Use Disorders Identification Test (AUDIT): WHO collaborative project on early detection of persons with harmful alcohol consumption. II. Addiction 1993;88:791-804.

15. Rabin R, Charro F. EQ-5D: a measure of health status from the Euroqol group. Ann Med 2001;33:337-43.

16. Miller WR, Rollnick S. II colloquio motivazionale. Preparare la persona al cambiamento, Erickson, 2004. 